\title{
Number magnitude orients attention, but not against one's will
}

\author{
GIOVANNI GALFANO \\ Università di Padova, Padua, Italy \\ ELENA RUSCONI \\ University College London, London, England \\ and \\ CARLO UMILTÀ \\ Università di Padova, Padua, Italy
}

\begin{abstract}
Recent evidence has shown that uninformative numbers can trigger attention shifts congruent with the spatial representation of number magnitude (Fischer, Castel, Dodd, \& Pratt, 2003). In the present study, three spatial-cuing experiments whose aim was to qualify the automaticity of this numbermediated orienting are described. Experiment 1 replicated the phenomenon, showing that uninformative numbers can evoke orienting in a simple detection task. In Experiment 2, target location was random, but the participants were encouraged to shift attention to the left in response to large numbers and to the right in response to small numbers. No evidence for strong automaticity was observed, since the participants' performance was better when left-side targets were preceded by large numbers than when they were preceded by small numbers and vice versa. Experiment 3 corroborated this pattern by comparing gaze- and number-mediated cuing under conditions of real counterpredictiveness. The results indicate that unlike gaze-driven orienting, number-mediated orienting is not obligatory.
\end{abstract}

Visuospatial attention orienting has been investigated by presenting cues such as central informative arrows or peripheral uninformative abrupt onsets (e.g., Jonides, 1981). Through these signals, two distinct modes of attentional control have been identified: an exogenous and an endogenous mode. In recent years, the dichotomy between goal-directed orienting mediated via central informative symbolic cues and stimulus-driven orienting mediated by peripheral abrupt onsets has been challenged by studies highlighting that (1) a number of cues with high biological relevance, such as eye gaze and body shadows, generate attention shifts whose features do not conform to the dichotomy (e.g., Friesen \& Kingstone, 1998; Galfano \& Pavani, 2005), and (2) centrally presented symbolic cues can orient attention even when they are uninformative (e.g., Hommel, Pratt, Colzato, \& Godijn, 2001).

Fischer, Castel, Dodd, and Pratt (2003) tested whether similar effects can be obtained using numbers as (unin-

Part of the results reported here were presented at the joint meeting of the Experimental Psychology Society and the Canadian Society for Brain, Behaviour, and Cognitive Science, July 2005, Montreal. This research was supported in part by grants from MIUR (Ministero dell'Istruzione, dell'Università e della Ricerca) and the University of Padua to C.U. We are grateful to Luca Antonello and Laura Seccafien for collecting the data and to Cathleen Moore, Alan Kingstone, and two anonymous reviewers for their insightful suggestions. Correspondence concerning this article should be addressed to G. Galfano, Dipartimento di Psicologia dello Sviluppo e della Socializzazione, Università di Padova, via Venezia, 8, 35131-I, Padua, Italy (e-mail: giovanni.galfano@unipd.it). formative) cues. This idea was inspired by the notion of a mental number line (MNL) representing numbers from left to right (Restle, 1970). Fischer et al. presented the numbers $1,2,8$, and 9 at fixation. After different stimulus onset asynchronies (SOAs), a target requiring a simple detection response appeared in one of two peripheral boxes. The target had the same probability to appear in the left or the right box, irrespective of number magnitude, and the participants knew that the numbers were task irrelevant. If mere observation of numbers activated their position on the MNL, one might expect either a left or a right attentional shift, depending on number magnitude. The results showed that the participants were, indeed, faster when targets appearing on the left box were preceded by small numbers and when targets appearing on the right box were preceded by large numbers. Because numbers were uncorrelated with target location, Fischer et al. concluded that merely viewing numbers evokes automatic magnitudedependent attentional shifts.

The present study assessed the degree of automaticity of number-mediated attentional shifts. According to the intentionality criterion (Jonides, 1981), orienting of attention is automatic if it resists suppression and occurs regardless of participants' expectations. In Fischer et al.'s (2003) study, numbers were task irrelevant; thus, it seems reasonable to conclude that they evoked an involuntary shift of attention, because the participants had no incentive for it to happen. However, to conclude that numbers elicited an obligatory shift of attention, it is necessary to 
show that this occurred even when participants intended for it not to happen.

In Experiment 1, we established a baseline condition by replicating Fischer et al.'s (2003) findings. Experiments 2 and 3 tested the criterion of intentionality for numbermediated attention shifts by manipulating expectancies.

\section{EXPERIMENT 1}

The experimental setting was identical to that in Fischer et al. (2003), with three exceptions. First, we used only two SOAs (500 and $800 \mathrm{msec}$ ), in order to increase the data points for each relevant condition. The shorter SOA did not produce reliable effects in Fischer et al., whereas the longer revealed the strongest effect. Second, we included the digit 5 among the cue numbers, to implicitly provide the participants with a landmark of the middle point among the other cue numbers $(1,2,8$, and 9$)$. Third, following studies on eye gaze cues (e.g., Friesen, Ristic, \& Kingstone, 2004), no interstimulus interval was present between cue offset and target onset, which resulted in a longer cue duration, in comparison with that in Fischer et al.'s study, without changing the overall SOA duration. The latter changes had the purpose of maximizing the activation of a left-right dimension in the MNL, which, in turn, should increase the likelihood of number-mediated attention shifts.

\section{Method}

Participants. Twenty-six undergraduate students with normal or corrected-to-normal vision participated in the experiment.

Apparatus, Stimuli, and Procedure. A Pentium III PC was used for stimulus timing and response collection. The participants sat $50 \mathrm{~cm}$ from a 17 -in. monitor $(1,024 \times 768,85 \mathrm{~Hz})$, with their head on a chinrest. The stimuli appeared in white against a black background. A fixation cross $(1 \times 1 \mathrm{~mm})$ was presented in the center of the screen, along with two symmetrically positioned boxes $(3 \times 3 \mathrm{~cm})$ aligned with the horizontal meridian. Their inner edges were located $10 \mathrm{~cm}$ to the left and right from the center of the screen. The cue $(1-\mathrm{cm}$ width $\times$ 1.7-cm height) was one of five possible digits $(1,2,5,8$, or 9$)$ replacing the fixation point with the same probability. Digits were set in 42-point Arial bold font. The target was a filled circle ( $7 \mathrm{~mm}$ of radius) centered on the left or right box equally often. Each trial started with the onset of the boxes along with the fixation cross (see Figure 1A). After $500 \mathrm{msec}$, one of the five digits appeared, replacing the fixation cross. After 500 or $800 \mathrm{msec}$, the target appeared in one of the boxes, while the fixation cross replaced the cue. This display remained visible until a response was made or $1,000 \mathrm{msec}$ had elapsed. The participants performed a simple detection task by pressing the space bar with their preferred hand. They were instructed to keep their eyes at fixation throughout the trial and to respond as quickly as possible. Catch trials (where no target appeared) were also included ( $17 \%$ of the total trials). The intertrial interval was $1,000 \mathrm{msec}$.

Trials in which target location was spatially congruent with the location occupied by the cue number on the MNL were considered as valid trials. Conversely, invalid trials were those in which target location was spatially incongruent with the location of the cue. Trials in which the cue was a 5 were considered as neutral trials. ${ }^{1}$ The participants performed two blocks of 240 trials, with 40 catch trials per block. SOA was equiprobably 500 or $800 \mathrm{msec}$. For each SOA, there was an equal number of trials (50) with the target on the left and on the right box. In total, there were 20 trials for each cue number for each level of SOA and target location. This resulted in 80 valid trials, 80 invalid trials, and 40 neutral trials for each block. The participants knew that the cue provided no information about target location.
}

Prior to the experimental session, the participants performed 20 randomly chosen practice trials. The design comprised three withinparticipants factors: number magnitude (small, neutral, or large), target location (left or right), and SOA (500 or $800 \mathrm{msec}$ ).

\section{Results and Discussion}

Catch trial errors and missed responses were fewer than $2 \%$ and were not analyzed. Correct median reaction times (RTs) were submitted to a three-way repeated measures ANOVA. The main effect of target location was significant $\left[F(1,25)=6.05, M S_{\mathrm{e}}=994, p=.02\right]$, with shorter RTs for right targets $(310 \mathrm{msec})$ than for left targets $(318 \mathrm{msec}){ }^{2}$ The main effect of SOA was significant $\left[F(1,25)=8.09, M S_{\mathrm{e}}=\right.$ $704, p<.01$ ], since RTs were shorter at the 800-msec SOA than at the 500-msec SOA, likely reflecting a foreperiod effect. The number magnitude $\times$ target location interaction was significant $\left[F(2,50)=5.39, M S_{\mathrm{e}}=156, p=.007\right]$, whereas the SOA $\times$ number magnitude $\times$ target location interaction was not $\left[F(2,50)=0.77, M S_{\mathrm{e}}=193, p=.46\right]$. Planned comparisons (one-tailed $t$ tests) revealed that the participants were significantly faster when a left-sided target was preceded by a small number than when it was preceded by a large number $[t(25)=1.75, p=.04$; see Tables $1-3]$. Similarly, the participants were significantly faster when a right-sided target was preceded by a large number than when it was preceded by a small number $[t(25)=1.59, p=.04]$. No other comparisons were significant.

Neutral trials did not reveal costs or benefits. However, although the effect of numbers on attention shifts was small $(6.5 \mathrm{msec}),{ }^{3} \mathrm{RTs}$ associated with neutral trials were in the expected direction (see Table 1). The basic effect in Fischer et al. (2003) was replicated, although no modulation was observed as a function of SOA. One aspect that might have played a role is that we kept the number visible for the whole SOA duration, which might have promoted better stimulus encoding and, therefore, faster activation of the MNL.

In the next experiments, we assessed the automaticity of number-driven attention shifts by testing the intentionality criterion.

\section{EXPERIMENT 2}

In the present experiment, we replicated Experiment 1, with one important difference. Although the proportion of valid and invalid trials was the same as in Experiment 1,

Table 1

Mean Median Reaction Times (RTs, in Milliseconds) of Correct Responses to Left and Right Targets as a Function of Cue Number Magnitude in Experiments 1 and 2

\begin{tabular}{|c|c|c|c|c|c|}
\hline & \multirow{3}{*}{$\begin{array}{l}\text { Number } \\
\text { Magnitude }\end{array}$} & \multicolumn{4}{|c|}{ Target Location } \\
\hline & & \multicolumn{2}{|c|}{ Left } & \multicolumn{2}{|c|}{ Right } \\
\hline & & $M$ & $S E$ & $M$ & $S E$ \\
\hline \multirow[t]{3}{*}{ Experiment 1} & Small & 314 & 10 & 313 & 9 \\
\hline & Neutral & 319 & 11 & 310 & 9 \\
\hline & Large & 320 & 10 & 306 & 10 \\
\hline \multirow[t]{3}{*}{ Experiment 2} & Small & 358 & 11 & 346 & 11 \\
\hline & Neutral & 354 & 11 & 347 & 11 \\
\hline & Large & 352 & 10 & 352 & 11 \\
\hline
\end{tabular}




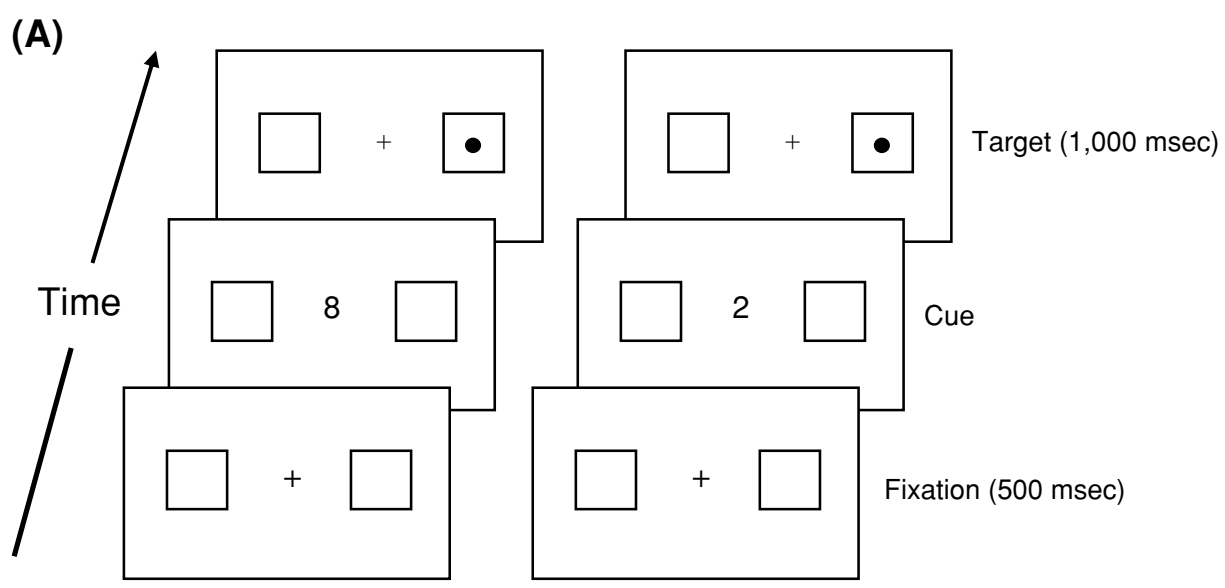

(B)
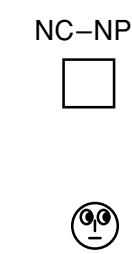

Predicted

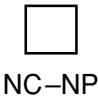

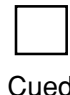

Cued

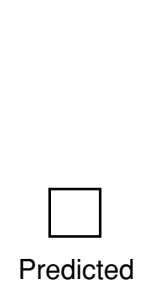

Predicted

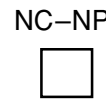

9

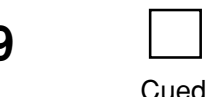

Cued

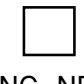

$\mathrm{NC}-\mathrm{NP}$

Figure 1. (A) Sequence of events in Experiments 1 and 2. A valid trial (on the left) and an invalid trial (on the right) are shown. Valid trials were those in which target location in the physical space was spatially congruent with the location occupied by the cue number in the representational space. Conversely, invalid trials were trials in which target location in the physical space was spatially incongruent with the location occupied by the cue number in the representational space. The stimuli are not drawn to scale. Cue duration (SOA) was 500 or 800 msec in Experiment 1. In Experiment 2, a longer (1,100 msec) cue duration was also included. (B) Example of displays for the gaze (on the left) and number (on the right) cue blocks in Experiment 3. Cue duration (SOA) was 600 msec. Cues were genuinely counterpredictive as to target location. The stimuli are not drawn to scale. Predicted, target occurs at the predicted (noncued) location; cued, target occurs at the cued (not-predicted) location; NP-NC, target occurs at a location that is neither cued nor predicted.

the participants were told that the target was more likely to appear inside the left box after a large number and inside the right box after a small number. Thus, they were encouraged to shift attention leftward after viewing a large number and rightward after viewing a small number. The number 5 provided no information as to target location. The manipulation introduced in the present experiment rendered the cue a task-relevant stimulus, since the participants were explicitly asked to process it. Because the relation between number magnitude and target location was made explicit in the instructions, this should have strengthened the activation of number magnitude, increasing the chance of attention shifts compatible with the MNL. In sum, in the present experiment, endogenous and possible exogenous orienting mechanisms were set against each other. If the participants were unable to prevent involuntary orienting to the location of a number in cognitive space, we would expect to find an effect similar to that observed in Experiment 1, at least at the shortest SOA. By contrast, a reversed pattern would suggest that the participants had efficiently inhibited involuntary orienting when they believed that it would have been detrimental to performance on most trials.

\section{Method}

Participants. Twenty-four undergraduates with normal or corrected-to-normal vision participated in the experiment.

Stimuli and Procedure. The same apparatus and stimuli as those in Experiment 1 were adopted. An additional SOA of 1,100 msec was used, since we hypothesized that voluntary orienting might take time to develop, being secondary to the processing of number magnitude. The participants undertook four blocks of 180 trials. In each block, there were 30 catch trials. The SOA was equiprobably 500 , 800 , or $1,100 \mathrm{msec}$. In total, there were 20 trials for each cue number for each level of SOA and target location. Before the experimental session began, the participants were instructed to shift attention leftward after viewing a large number and rightward after viewing a small number, since number magnitude was counterpredictive as 
to target location. However, as in Experiment 1, target location was uncorrelated with number magnitude.

\section{Results and Discussion}

Catch trial errors and missed responses were fewer than $1 \%$ and were not analyzed. Correct median RTs were submitted to a three-way repeated measures ANOVA with the same factors as those in the previous experiment. The main effect of target location was significant $[F(1,23)=$ $\left.8.49, M S_{\mathrm{e}}=392, p<.01\right]$, revealing shorter RTs for right targets $(348 \mathrm{msec})$ than for left targets $(354 \mathrm{msec})$. The main effect of SOA was also significant $[F(2,46)=$ $\left.35.82, M S_{\mathrm{e}}=639, p<.01\right]$, due to shorter RTs at the 1,100- and 800-msec SOAs than at the 500-msec SOA. The number magnitude $\times$ target location interaction was significant $\left[F(2,46)=5.42, M S_{\mathrm{e}}=366, p<.01\right]$, whereas the SOA $\times$ number magnitude $\times$ target location interaction was not $\left[F(4,92)=0.46, M S_{\mathrm{e}}=209, p=.76\right]$. Planned comparisons revealed that the participants were significantly faster when a left-sided target was preceded by a large number than when it was preceded by a small number $[t(23)=2.94, p=.01$; see Tables $1-3]$. Also, the participants were significantly faster when a right-sided target was preceded by a small number than when it was preceded by a large number $[t(23)=2.17, p=.02]$. No other comparisons were significant.

Number-mediated orienting of attention was reversed here, when the participants were encouraged to orient toward the box opposite to that suggested by number magnitude. This indicates that participants can successfully counteract the involuntary orienting response induced by number magnitude. The claim is supported by a comparison of the validity effect at the 500- and 800-msec SOAs for Experiments 1 and 2, using a four-way betweenparticipants ANOVA with the additional factor of experiment. The experiment $\times$ number magnitude $\times$ target lo-

Table 2

Mean Median Reaction Times (RTs, in Milliseconds) of Correct Responses to Left and Right Targets as a Function of Stimulus Onset Asynchrony (SOA) and Cue Number Magnitude in Experiments 1 and 2

\begin{tabular}{|c|c|c|c|c|c|c|}
\hline & \multirow{3}{*}{$\begin{array}{c}\text { SOA } \\
(\mathrm{msec})\end{array}$} & \multirow{3}{*}{$\begin{array}{c}\text { Number } \\
\text { Magnitude }\end{array}$} & \multicolumn{4}{|c|}{ Target Location } \\
\hline & & & \multicolumn{2}{|c|}{ Left } & \multicolumn{2}{|c|}{ Right } \\
\hline & & & $M$ & $S E$ & $M$ & $S E$ \\
\hline \multirow[t]{6}{*}{ Experiment 1} & 500 & Small & 315 & 10 & 320 & 9 \\
\hline & & Neutral & 326 & 11 & 315 & 9 \\
\hline & & Large & 322 & 10 & 311 & 10 \\
\hline & 800 & Small & 312 & 12 & 308 & 12 \\
\hline & & Neutral & 316 & 12 & 303 & 11 \\
\hline & & Large & 316 & 10 & 304 & 10 \\
\hline \multirow[t]{9}{*}{ Experiment 2} & 500 & Small & 370 & 11 & 360 & 11 \\
\hline & & Neutral & 369 & 11 & 359 & 10 \\
\hline & & Large & 366 & 10 & 365 & 11 \\
\hline & 800 & Small & 346 & 10 & 332 & 10 \\
\hline & & Neutral & 343 & 11 & 338 & 12 \\
\hline & & Large & 338 & 10 & 343 & 11 \\
\hline & 1,100 & Small & 357 & 11 & 345 & 12 \\
\hline & & Neutral & 351 & 11 & 344 & 12 \\
\hline & & Large & 344 & 11 & 348 & 11 \\
\hline
\end{tabular}

cation interaction was significant $\left[F(2,96)=7.93, M S_{\mathrm{e}}=\right.$ 223, $p<.01]$. Planned comparisons confirmed that RTs for detecting left and right targets showed an opposite pattern as a function of number magnitude in the two experiments. SOA was not involved in any significant interaction (all $F \mathrm{~s}<1$ ).

The results suggest that number-mediated orienting fails to fulfill the intentionality criterion. Crucially, cues such as eye gaze have proved to fulfill this criterion (Friesen et al., 2004). In the next experiment, we tested sensitivity to expectancy of number-driven orienting through a comparison with eye gaze cuing in the same participants, under conditions of real counterpredictiveness.

\section{EXPERIMENT 3}

In the present experiment, the participants were instructed to shift attention leftward after viewing a large number and rightward after a small number, but unlike in Experiment 2, information conveyed by the cue was counterpredictive as to target location. Friesen et al. (2004) have proved that eye gaze cuing is unaffected by manipulation of expectancies, since both expectancydriven (endogenous) and gaze-driven (exogenous) effects are observed, at least with a $600-\mathrm{msec}$ SOA. This result demonstrates that gaze-driven orienting not only meets the intentionality criterion (Jonides, 1981) but also is independent of endogenous orienting. In the present experiment, we compared how number and gaze direct attention, by rendering the targets more likely to appear in the location opposite to that indicated by the cue. SOA was fixed at $600 \mathrm{msec}$. Following Friesen et al., we used four, rather than two, possible target locations and compared the participants' performance in cued trials (targets appearing in a location that was either gazed-at or congruent with MNL), predicted trials (targets appearing in the most likely location-i.e., opposite to either gazed-at locations or incongruent with MNL, along the same meridian), and noncued-nonpredicted trials (NC-NP-i.e., targets appearing in a location positioned along the meridian opposite to the one that had been cued). Friesen et al.'s experimental setting was slightly modified, because directional meaning could be conveyed by four cues for gaze (a face looking to the box located to the left, right, top, or bottom), whereas the same cues may cause attention shifts along both the horizontal and the vertical meridians in the case of numbers. Furthermore, Ristic and Kingstone (2006) have shown that, when uninformative numbers are used, adding a top target location and a bottom target location is sufficient to abolish the left-to-right MNL effect (likely because left-right are no longer assigned a special status, relative to top-bottom).

Thus, the participants knew that if they were shown a leftward gaze, the target was more likely (75\% of the trials) to appear in the right box (predicted trials) and that, in the remaining trials, the target appeared with the same probability in the left box (cued trials), in the bottom box, or in the top box (NC-NP trials). Similarly, the participants knew that if they were shown a small (i.e., 1 or 2 ) 
Table 3

Mean Median Reaction Times (RTs, in Milliseconds) of Correct Responses to Left and Right Targets as a Function of Individual Cue Number in Experiments 1 and 2

\begin{tabular}{|c|c|c|c|c|c|}
\hline & \multirow[b]{3}{*}{ Cue Number } & \multicolumn{4}{|c|}{ Target Location } \\
\hline & & \multicolumn{2}{|c|}{ Left } & \multicolumn{2}{|c|}{ Right } \\
\hline & & $M$ & $S E$ & $M$ & $S E$ \\
\hline \multirow[t]{5}{*}{ Experiment 1} & 1 & 314 & 11 & 311 & 9 \\
\hline & 2 & 314 & 10 & 315 & 10 \\
\hline & 5 & 319 & 11 & 310 & 9 \\
\hline & 8 & 321 & 10 & 307 & 10 \\
\hline & 9 & 319 & 10 & 305 & 10 \\
\hline \multirow[t]{5}{*}{ Experiment 2} & 1 & 359 & 11 & 347 & 11 \\
\hline & 2 & 358 & 10 & 346 & 11 \\
\hline & 5 & 354 & 11 & 347 & 11 \\
\hline & 8 & 353 & 10 & 354 & 11 \\
\hline & 9 & 350 & 11 & 350 & 10 \\
\hline
\end{tabular}

number, the target was more likely ( $75 \%$ of the trials) to appear in the right box (predicted trials) and that, in the remaining trials, the target appeared with the same probability in the left box (cued trials), in the bottom box, or in the top box (NC-NP trials). The same happened for rightward gaze and large numbers.

This procedure induced a bias to shift attention along the horizontal meridian (target appeared in the left or right box on $80 \%$ of target-present trials), which should increase the possibility, if any, to observe a number-driven orienting effect (Ristic \& Kingstone, 2006). Note that the crucial comparisons (cued vs. NC-NP, predicted vs. NC-NP) for assessing gaze- and number-driven shifts involved trials on which target location was different (the target appeared always along the vertical meridian in NC-NP trials and always along the horizontal meridian in cued and predicted trials). Therefore, we included a neutral condition, to rule out the possibility that significant differences in the crucial comparisons could be accounted for by differences in the target location. In the neutral condition, the target appeared along the vertical as likely as the horizontal meridian and was preceded by a straight gaze or the number 5 . If target location did not exert a systematic influence on trial type, no significant differences in neutral trials as a function of whether the target appeared along the vertical or the horizontal meridian would be expected. ${ }^{4}$

Having used a 600-msec SOA, for gaze we predicted worse performance on NC-NP trials than on both predicted and cued trials, thus replicating the pattern in Friesen et al. (2004). By contrast, for numbers, on the basis of Experiment 2, we expected only a significant effect of expectancies (i.e., shorter RTs for predicted than for NC-NP trials).

\section{Method}

Participants. Twenty undergraduates participated. All reported normal or corrected-to-normal vision.

Stimuli and Procedure. The apparatus and stimuli were the same as those in the previous experiments. However, two boxes were added along the vertical meridian, having the same distance from fixation as those placed along the horizontal meridian. Cue type varied across blocks. The procedure for the number blocks was the same as that in the previous experiments. Eye gaze cues (see Figure 1B) consisted of a black line drawing of a face on a white background. The round face outline $(1.2 \mathrm{~cm}$ of diameter) contained two circles (the eyes, $4 \mathrm{~mm}$ of diameter) and two straight lines (nose and mouth, $4 \mathrm{~mm}$ in length and in width, respectively) and was centered on the screen. The center of each eye was located $2.5 \mathrm{~mm}$ above and $2.5 \mathrm{~mm}$ left and right of the center of the screen. The nose was aligned with the center of the screen along the vertical meridian. The mouth was centered $2.5 \mathrm{~mm}$ below the center of the screen. The pupils (black filled circles, $2 \mathrm{~mm}$ of diameter) appeared together with the face. For straight gaze trials, they were centered both vertically and horizontally in the eyes, whereas for left and right gaze, they were just touching the eyes either leftward or rightward. Targets appearing in the top or bottom box were centered along the vertical meridian. The SOA was fixed at $600 \mathrm{msec}$.

The participants undertook two blocks of 270 trials for each cue type (gaze vs. number). Order was counterbalanced across participants. Each block comprised 40 neutral trials (10 for each target location), 150 predicted trials ( 75 for targets in the left box and 75 for targets in the right box), 10 cued trials ( 5 for targets in the left box and 5 for targets in the right box), $20 \mathrm{NC}-\mathrm{NP}$ trials ( 10 for targets in the top box and 10 for targets in the bottom box), and 50 catch trials. The participants were encouraged to shift attention leftward after viewing a large number (or a face gazing right) and rightward after viewing a small number (or a face gazing left).

\section{Results and Discussion}

False alarms and misses were fewer than $2 \%$ and were not analyzed. First, we asked whether performance was different if the target appeared along the vertical or the horizontal meridian on a neutral trial. This was necessary to rule out possible confounds in the test for exogenous and endogenous orienting. Hence, correct median RTs for neutral trials were submitted to a two-way repeated measures ANOVA with cue type (number or gaze) and target meridian (vertical or horizontal) as factors. No sources of variance were significant (lower $p=.25$ ), which suggests that the participants' performance was the same whether the target appeared along the vertical $(335 \mathrm{msec})$ or the horizontal (338 msec) meridian.

A second two-way repeated measures ANOVA was conducted with cue type (number or gaze) and trial type (predicted, cued, or NC-NP) as factors. Both cue type $\left[F(1,19)=5.18, M S_{\mathrm{e}}=893, p<.05\right]$ and trial type $\left[F(2,38)=40.38, M S_{\mathrm{e}}=3,934, p<.001\right]$ were significant. More crucially, also the cue type $\times$ trial type interaction was significant $\left[F(2,38)=6.42, M S_{\mathrm{e}}=521, p<\right.$ $.01]$. Planned comparisons revealed that the participants were faster on predicted than on NC-NP trials for both number $[t(19)=6.92, p<.01]$ and eye gaze $[t(19)=$ $6.01, p<.01]$ cues, which indicates that they relied on expectancies. In addition, the participants were significantly faster on cued trials than on NC-NP trials for gaze cues $[t(19)=1.79, p<.05]$, but not for number cues $(p=.40)$. This suggests that gaze elicits both endogenous and exogenous orienting, whereas numbers evoke only an expectancy-related attention shift (see Table 4). These results are consistent with those in Friesen et al. (2004) and show that, unlike gaze-driven orienting, number-mediated orienting fails to meet the intentionality criterion. 
Table 4

Mean Median Reaction Times (RTs, in Milliseconds) of Correct Responses for Gaze and Number Cues as a Function of Trial Type (Predicted, Cued, or NP-NC) in Experiment 3

\begin{tabular}{lccccc}
\hline & \multicolumn{3}{c}{ Cue Type } \\
\cline { 2 - 5 } & \multicolumn{2}{c}{ Gaze } & & \multicolumn{2}{c}{ Number } \\
\cline { 2 - 5 } \cline { 4 - 5 } Trial Type & $M$ & $S E$ & & $M$ & $S E$ \\
\hline Predicted & 289 & 13 & & 284 & 14 \\
Cued & 378 & 20 & 409 & 16 \\
NP-NC & 392 & 20 & 402 & 19 \\
\hline
\end{tabular}

Note-Predicted, target occurs at the predicted (noncued) location. Cued, target occurs at the cued (nonpredicted) location. NP-NC, target occurs at a location that is neither cued nor predicted.

\section{GENERAL DISCUSSION}

Experiment 1 replicated the basic effect reported by Fischer et al. (2003), showing that the participants oriented leftward after viewing a small number and rightward after a large number, even when they knew that numbers did not predict target location. Experiments 2 and 3 assessed the automaticity of this effect with reference to the intentionality criterion (Jonides, 1981). Experiment 2 showed that number-mediated orienting can be prevented by telling participants that number magnitude is counterpredictive with respect to target location, even when this, in fact, is random. One may argue that evidence for obligatory processing might have emerged if we had used shorter SOAs. However, according to Fischer et al., number-mediated orienting was not reliable for SOAs shorter than $700 \mathrm{msec}$. In Experiment 3, we contrasted the ability to evoke attention shifts exerted by eye gaze and numbers, using a $600-\mathrm{msec}$ SOA. In accordance with Friesen et al. (2004), we showed that eye gaze elicits independent expectancy-related and gaze-driven attention shifts, whereas numbers fail to elicit an obligatory orienting response (see also Ristic, Wright, \& Kingstone, 2006).

Taken together, our findings show that attention is not shifted according to the position occupied by a given number on the MNL against participants' intentions. Because numbers modulated performance only when the participants had no particular incentive for it to occur, we conclude that number-mediated attentional orienting is not obligatory but only stimulus driven. The fact that number-mediated orienting does not satisfy criteria for obligatoriness can be explained in terms of a hierarchy based on biological relevance reflected by different cues, with eye gaze and abrupt onsets situated at the highest level, followed by stimuli for which relevance is less hardwired and the left-right polarity is less fixed, being dependent on communication-based conventions (Hommel et al., 2001). Note that the left-right polarity in the MNL is not absolute, because it depends on the range of the numbers included in a given study. We believe this could explain why number-mediated orienting is not obligatory and seems to produce much weaker effects, in comparison with other cues, such as eye-gaze, that possess a higher biological value and convey a fixed, unambiguous, directional meaning.

\section{REFERENCES}

Fischer, M. H., Castel, A. D., Dodd, M. D., \& Pratt, J. (2003). Perceiving numbers causes spatial shifts of attention. Nature Neuroscience, 6, 555-556.

Friesen, C. K., \& Kingstone, A. (1998). The eyes have it! Reflexive orienting is triggered by nonpredictive gaze. Psychonomic Bulletin \& Review, 5, 490-495.

Friesen, C. K., Ristic, J., \& Kingstone, A. (2004). Attentional effects of counterpredictive gaze and arrow cues. Journal of Experimental Psychology: Human Perception \& Performance, 30, 319-329.

Galfano, G., \& PAVANI, F. (2005). Long-lasting capture of tactile attention by body shadows. Experimental Brain Research, 166, 518-527.

Hommel, B., Pratt, J., Colzato, L., \& Godijn, R. (2001). Symbolic control of visual attention. Psychological Science, 12, 360-365.

JoNIDES, J. (1981). Voluntary versus automatic control over the mind's eye's movement. In J. [B.] Long \& A. [D.] Baddeley (Eds.), Attention and performance $I X$ (pp. 187-203). Hillsdale, NJ: Erlbaum.

JonidEs, J., \& MACK, R. (1984). On the cost and benefit of cost and benefit. Psychological Bulletin, 96, 29-44.

RESTLE, F. (1970). Speed of adding and comparing numbers. Journal of Experimental Psychology, 83, 274-278.

Ristic, J., \& Kingstone, A. (2006). Attention to arrows: Pointing to a new direction. Quarterly Journal of Experimental Psychology, 59, 1921-1930.

Ristic, J., Wright, A., \& Kingstone, A. (2006). The number line effect reflects top-down control. Psychonomic Bulletin \& Review, 13, $862-868$.

\section{NOTES}

1. It has been known since Jonides and Mack (1984) that "neutral" cues can be problematic. Thus, although we incorporated in the analyses trials on which the target, when present, was preceded by the number 5 , their purpose was only to increase the likelihood that the MNL would be activated.

2. This pattern may be interpreted as evidence of asymmetric attention mechanisms in the two hemispheres. Alternatively, given that most of the participants were right-handed, it might reflect a spatial stimulusresponse compatibility effect.

3. The effect reported by Fischer et al. (2003) was approximately $10 \mathrm{msec}$ (estimated from Figure 1 in Fischer et al., 2003).

4. In accordance with Friesen et al. (2004), "neutral" trials were not included in the analyses for assessing the presence of gaze- and numberdriven orienting, to avoid confounds related to the comparison of performance across different cue conditions. Performance on "neutral" trials was important only for rejecting the possibility that differences in attention shifts could be accounted for by trivial differences in target processing along the vertical and horizontal meridians.

(Manuscript received May 24, 2005; revision accepted for publication February 13, 2006.) 